\title{
Segmentation of cells from 3-D confocal images of live embryo
}

\author{
Ali Zeynali Aaq Qaleh" ${ }^{1,}$, Seyyed Mahdi Haji Mirahmadi \\ ${ }^{1}$ Faculty of Engineering, Islamic Azad University, Qom, Iran \\ ${ }^{2}$ Software Engineer, Young Researchers and Elite Club, Qazvin Branch, Islamic Azad University, Qazvin, Iran
}

Email address:

a.zaynali.a@mail.com (A. Z. A. Qaleh), Caspiansys@gmail.com (S. M. H. Mirahmadi)

To cite this article:

Ali Zeynali Aaq Qaleh, Seyyed Mahdi Haji Mirahmadi. Segmentation of Cells from 3-D Confocal Images of Live Embryo. International Journal of Intelligent Information Systems. Special Issue: Research and Practices in Information Systems and Technologies in Developing Countries. Vol. 3, No. 6-1, 2014, pp. 45-48. doi: 10.11648/j.ijiis.s.2014030601.18

\begin{abstract}
Partial-differential-equation- based segmentation has been employed to accurately extract the shapes of membranes and nuclei from time lapse confocal microscopy images, taken throughout early Zebrafish embryogenesis. This strategy is a prerequisite for an accurate quantitative analysis of cell shape and morphodynamics during organogenesis and is the basis for an integrated understanding of biological processes. This data will also serve for the measurement of the variability between individuals in a population. The segmentation of cellular structures is achieved by first using an edge-preserving image filtering method for noise reduction and then applying an algorithm for cell shape reconstruction based on the Subjective Surfaces technique.
\end{abstract}

Keywords: Partial Differential Equations, Segmentation, Confocal Images, Morphodynamics

\section{Introduction}

The extraction and segmentation of true 3-D shapes is a crucial task in the analysis of morphodynamical patterns in biology. The shape reconstruction of nuclei and membranes during embryogenesis is the basis for a strategy of automated measurements of the cell proliferation rate in the embryonic tissues that will be used for designing low-cost methods for the pre-clinical evaluation of anti-cancer drug effects in vivo. Prior and during cell division, the cell shape undergoes characteristic changes. Thus, shape analysis of nuclei and membranes is essential for the detection of cell division which is necessary to get a measurement of the cell proliferation rate in living tissues. Furthermore, the reconstruction of the cellular shape will provide relevant parameters to mea- sure the variability between different individuals of the same species, opening the way for understanding the individual susceptibility to genetic diseases or response to treatments.

Our aim is to design an algorithm providing in an automated way the correct segmentation of nucl ei and membranes in live embryos. We expect to avoid the need for any manual intervention which is in any case completely unrealistic when dealing with thousands of objects. A similar study can be found in a previous work by Sarti et al. [1], where confocal microscopy images were processed to extract the shape of nuclei. However, in that case, the analyzed volumes were not acquired from a living organism but from pieces of fixed tissues.

\section{Imaging Acquisition}
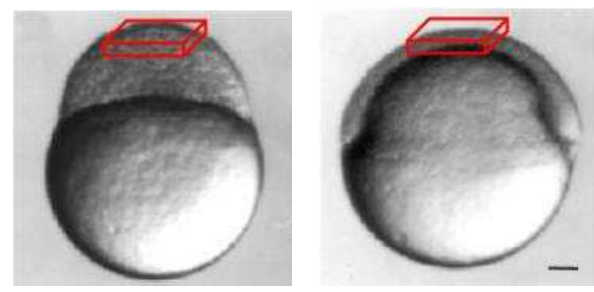

Fig1. Image of the Embryo.(Left) Start point (about 3 hours post fertilization).(Right) End point (about 7 hours post fertilization).

$$
\Phi_{t}={ }_{g} H|\nabla \Phi|+\nabla_{g} . \nabla \Phi ; \quad H=\nabla \cdot\left(\frac{\nabla \Phi}{|\nabla \Phi|}\right)
$$

First To obtain accurate measurements of 3-D features at the cellular level in living embryos, it is necessary to use an acquisition technique with micrometrical resolution and to reconstruct volumetric information. To fulfil these requirements, the analyzed images have been acquired by 
confocal microscopy with the best compromise in terms of spatial and temporal resolution [2].

In order to produce high contrast images, the specimen has been labeled through the expression of fluorescent proteins, the eGFP (Green Fluorescent Protein, targeted to the nuclei) and the mcherry (Red Fluorescent Protein, addressed to membranes). The two channels were acquired separately but simultaneously, as the emission spectrum of the two proteins are sufficiently distinct.

The $\mathrm{x}, \mathrm{y}$ size of the acquired images is 512 x 512 pixels; the temporal resolution is about 5 minutes. The voxel size is not uniform in space: $0.584793 \mu \mathrm{m}$ in $\mathrm{x}$ and $\mathrm{y}$ directions and $1.04892 \mu \mathrm{m}$ in $\mathrm{z}$. The overall volume submitted to optical sectioning is 30 microns thick. The embryo has been imaged from 3,5 hours post fertilization (development at $280 \mathrm{C}$ ) for 4 hours (25o $\mathrm{C}$ under the microscope) [3].

\section{Image Denoising}

The noise present in the image can disrupt the shape information, therefore the de-noising process is an essential preliminary task in images segmentation. Different sources of noise can be identified: the non-homogeneous concentration of the fluorescent proteins in the labelled structures and the electronic noise from the instrument. In order to accurately reconstruct the object shape, the de-noising process has to improve the signal-to-noise ratio, faithfully preserving the edges position and definition. The geodesic curvature filtering $[1,4]$ is able to achieve this task. In the uniform regions, it moves the iso-intensity surfaces in the normal direction with a curvature-dependent speed, smoothing the superimposed noise, whereas near the edges it attracts the image levels towards the local contours, sharpening the edges and working to preserve the objects dimensions.

\section{Segmentation}

\subsection{Theoretical Background}

Beside the Subjective Surfaces technique $[5,6]$ is particularly useful for the segmentation of incomplete contours, because it allows the reconstruction and the integration of lacking information. The analyzed images, especially the membranes images, are characterized by a signal which is almost undetectable or even absent in some regions. In such situations, the Subjective Surfaces technique should allow the completion of lacking-portions of objects.

Consider a 3-D image $\mathrm{I}:(\mathrm{x}, \mathrm{y}, \mathrm{z}) \rightarrow \mathrm{I}(\mathrm{x}, \mathrm{y}, \mathrm{z})$ as a real positive function defined in some domain $\mathrm{M} \subset \mathrm{R} 3$.

One initial task in image segmentation is to build an edge indicator $\mathrm{g}$, which is a representation of the local structures of the image. An expression of $g$ can be [7].

Where $\mathrm{G} \sigma(\mathrm{x}, \mathrm{y}, \mathrm{z})$ is a Gaussian kernel with standard deviation $\sigma,(*)$ denotes the convolution and $\mathrm{n}$ is 1 or 2 . The value of $\mathrm{g}$ is close to 1 in flat areas $(|\nabla \mathrm{I}| \rightarrow 0)$ and close to 0 where the image gradient is high (edges). Thus, the minima of $\mathrm{g}$ denote the position of edges and its gradient is a force field that can be used to drive the evolution, because it always points in the local edge direction. The second step is the selection of a reference point, approximately in the center of the object to be segmented. The initial hypersuface $\mathrm{Si}$ ( $\mathrm{Si}:$ (x, $\mathrm{y}, \mathrm{z}) \rightarrow(\mathrm{x}, \mathrm{y}, \mathrm{z}, \Phi 0))$ is defined in the same domain $\mathrm{M}$ of the image I starting from an initial function $\Phi 0$. There are some alternative forms for $\Phi 0$, for example $\Phi 0=-\alpha \mathrm{D}$ or $\Phi 0=\alpha / \mathrm{D}$, where $\mathrm{D}$ is the 3-D distance function from the reference point. The motion equation, which drives the hypersurface evolution, is the flow which ensures the steepest descent of the hypersurface volume:

where $\mathrm{H}$ is the Euclidean mean curvature. This equation is exactly the same of that defined in the well-known Geodesic Active Contours technique (GAC) [8], except for a parameter introduced in $\mathrm{H}$ expression to weigh the matching of level curves. The entire hypersurface is driven under a speed law dependent on the image gradient, whereas in classical formulation of Level Set methods, as in GAC, the evolution affects only a particular front or level. The first term on the right side of equation (1) is a parabolic motion that evolves the hypersurface in normal direction with a velocity weighted by the mean curvature and by the edge indicator $g$, slowing down near the edges (where $g \rightarrow 0$ ). The second term on the right is a pure passive advection along the velocity field $-\nabla g$ whose direction and strength depend on its position. This term attracts the hypersurface in the direction of the image edges. In regions with subjective contours, continuation of existing edge fragments is negligible and equation (1) can be approximated by a geodesic flow, allowing the boundary completion with curves of minimal length (i.e. straight lines).

\subsection{Numerical Approximation}

After The partial derivatives in equation (1) are approximated with finite differences [5, 6, and 9]. Let us consider a uniform grid in space-time $(t, x, y, z)$, then the grid consists of the points $(t n, x i, y j, z k)=(n \Delta t, i \Delta x, j \Delta y, k \Delta z)$.

$\Phi_{\mathrm{i}, \mathrm{j}, \mathrm{k}}^{\mathrm{n}}$ We denote with the value of the function $\Phi$ at the grid point ( $t n, x i, y j, z k)$. Time derivatives are discretized with first order forward differences, the parabolic term with central differences and the advective term with upwind schemes, where the direction of the one-sided difference used in a point depends on the direction of the velocity field $-\nabla g$ in the same point.

\subsection{Modification to the Algorithm for Cells Segmentation}

In this section, we briefly introduce the segmentation algorithm based on the Subjective Surfaces technique. It allows the extraction of all the membranes and nuclei in the acquired volumes, processing the two channels separately. The focus point for the segmentation of hundreds of cells is to achieve a fully automated procedure; therefore the algorithm has been implemented to completely avoid the user intervention.

Every object is segmented separately from the others, limiting the computation to subvolumes containing only one cell. A different function $\Phi 0$ is defined for every cell, starting 
from a reference point automatically detected and located roughly in the center of the object. The hypersurface evolves under the flow equation (1) and, at the end of every partial computation, all the segmented surfaces are collected in a single total result.

Membranes segmentation requires an additional preprocessing: membranes images are corrupted by a weak nuclei signal, more intense during mitosis, which has to be removed because it can cause a wrong interpretation of the edges. This is due to overlapping between nuclei and membranes emission range during acquisition. A preliminary thresholding of nuclei images separates the nuclei signal from the background, then the interfering signal is removed from membranes images.

The algorithm has been implemented using the programming language $\mathrm{C}++$ and libraries ITK [10] and VTK [11]. The final version has been integrated in a framework de- signed for managing series of 3-D biological images [12].

\subsection{Edge Detection}

The analyzed images (membranes versus nuclei) behave in a completely different way in terms of edge detection: The thickness of membranes signal is of about 3 or 4 voxels, whereas nuclei are solid objects. These specific features require using different functions for the detection of edges position in nuclei and membranes images.

In nuclei images, the contours to be segmented are located in the regions where image gradient is higher and the minima of (1) denote the position of the edges (Fig. 2(a)). On the contrary, the function (1) can't be applied on membranes images because it reveals a double contour, on the internal and the external side of the cell. An alternative edge indicator has been defined using the image itself (not its gradient) as contours detector. We can use the intensity information to locate the position of the edges, because the membranes images contain high intensity regions, where the labeled membrane structure has been acquired, versus low intensity background regions. The edge indicator we used is:

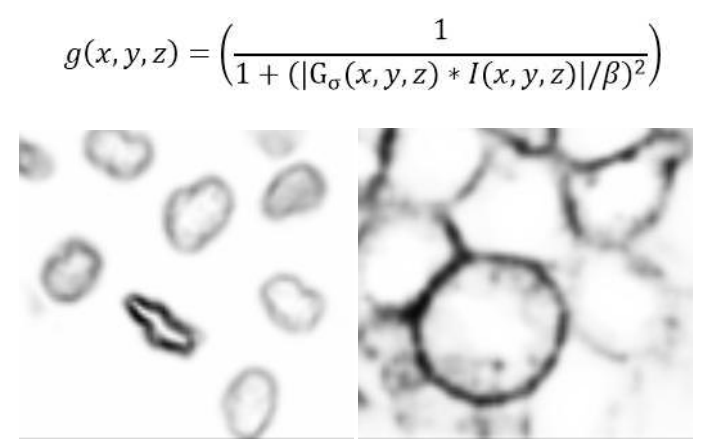

Fig 2. Images of the edge indicators: membranes (a) and nuclei (b).

As we expected, its minima locate the contours in the middle of the membranes thickness (Fig. 2(b)).

\subsection{Cells Recognition and Location}

A typical image contains a large number of cells, therefore the segmentation approach has to require minimal user intervention. It means that the interactive step of the Subjective Surfaces technique, that is the choice of the reference point inside the object to segment, should be automated. This goal is achieved with the generalized 3-D Hough Transform [13] that allows detecting specific shapes in the image. Approximating the nucleus as a spherical object, the Hough Transform is able to recognize every nucleus, returning its center [14]. This point, which is roughly the cell center in early embryonic cells, is the initial condition for the segmentation of nucleus and membrane belonging to the same cell.

\section{Results and Discussion}

We obtained good results assigning different weighting factors to the curvature and the advective motions, respectively first and second term on the right side of equation (1). The same expression of $S i$ can be employed both for nuclei and membranes segmentation. We used the initial function $\Phi 0=\alpha / D$, instead of $\Phi 0=-\alpha D$, to have an higher contrast in the processed image. After segmentation, the intensity distribution of the function $\Phi$ is typically associated to a bimodal histogram with a values range between 0 and 255 , because of a linear rescaling. The higher intensity peak (near to 255) corresponds to the segmented object, the lower one to the background. Therefore, the segmented surfaces can be extracted as the iso surfaces corresponding to the intermediate value 128 .

Fig. 3 shows the effect of boundary completion on membranes images: the missing contour, underlined by the red circle, is completed by a straight line. The algorithm shows the same behaviour for dividing membranes during telophase (Fig. 4), where there are two different nuclei inside the same cell and the membrane presents a constriction along the division plane. In this case, the algorithm segments two cells, because the Hough Transform detects two centers, and their contours are completed by straight lines.

The eye inspection of the resulting surfaces reveals some problems in the segmentation of the epithelial cells membranes. These cells surrounding the embryo are very flat.

This feature impairs membrane completion by the Subjective Surfaces 3-D technique, because the small extension in depth stops the evaluative process.

Before undergoing division, cells become spherical, whereas nuclei staining elongates as the chromosomes arrange in the future cell division plane (Fig. 5). It should be noted that the nucleus size is underestimated in the last two parts.
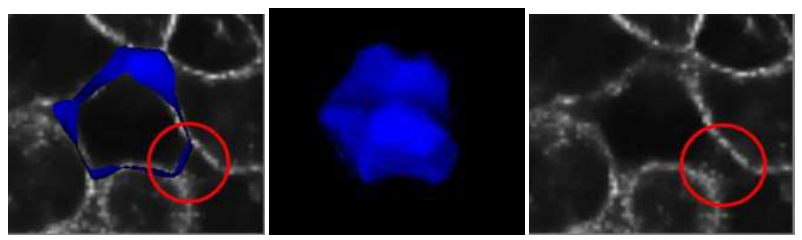

Fig 3. Segmentation of a membrane with an uncomplete contour. 

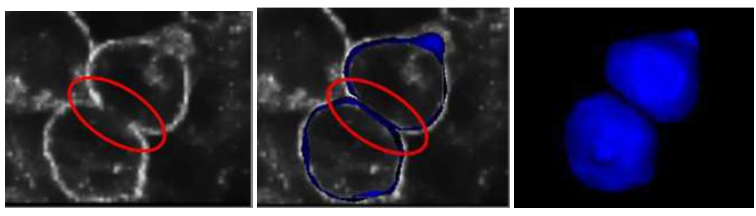

Fig 4. Segmentation of a dividing cell.
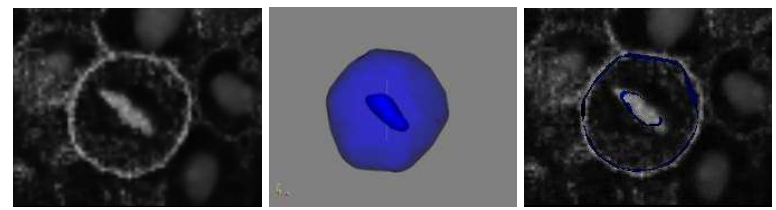

Fig 5. Segmentation of a dividing cell.

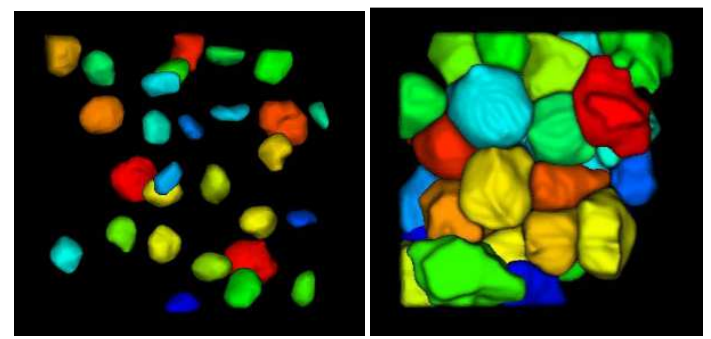

Fig 6. Segmentation of an entire subvolume.

This is due to the parabolic regularization term in the motion equation (1), which prevents the segmented surface to reach the contour if it is concave and with high curvature. However, the nuclei of not dividing cells are correctly segmented, as confirmed by visual inspection. Finally, in Fig. 6 we show the segmentation of two subvolumes of nuclei and membranes.

Visual inspection of the results has shown the ability of the algorithm to complete the missing contours, especially in membranes images, and to correctly reproduce the objects shape. The precision seems to decrease for elongated and flat shapes (epithelial cells and dividing nuclei).

The algorithm can be improved by integrating the segmentation of membranes and nuclei in the same process. An ad hoc method can be designed for the segmentation of the epithelial cells that have to be localized, prior segmentation, using a discriminating factor. Our segmentation procedure will now be tested on larger image data sets encompassing ten times of cells for a period of time at least twice as long. This should bring us close to an automated segmentation procedure for the whole zebrafish early embryogenesis.

\section{References}

[1] A. Sarti, C. O. de Solo'rzano, S. Lockett and R. Malladi, "A Geometric Model for 3-D Confocal Image Analysis", IEEE Transactions on Biomedical Engineering, vol. 47, pp. 1600-1609, 2000.

[2] S. Megason and S. Fraser,'Digitizing life at the level of the cell: highperformance laser-scanning microscopy and image analysis for in toto imaging of development," Mech. Dev., vol. 120, pp. 1407-1420, 2003.

[3] C. B. Kimmel, W. W. Ballard, S. R. Kimmel, B. Ullmann, and Th. F. Schilling, "Stages of embryonic development of the zebrafish," Dev. Dyn., vol. 203 , pp. 253-310, 1995.

[4] B. Rizzi., "3D Zebra Fish Embryo Images Filtering by Nonlinear Partial Differential Equations," 29th Annual International Conference of the IEEE Engineering in Medicine and Biology Society.

[5] A. Sarti, R. Malladi, and J. A. Sethian, "Subjective Surfaces: A Method for Completing Missing Boundaries," Proceedings of the National Academy of Sciences of the United States of America, vol. 12, pp.6258-6263, 2000.

[6] A. Sarti, R. Malladi, and J. A. Sethian,"Subjective Surfaces: A Geometric Model for Boundary Completion," International Journal of Computer Vision, vol. 46, pp. 201-221, 2002.

[7] P. Perona and J. Malik,"Scale-space and edge detection using anisotropic diffusion," IEEE Trans. Pattern Anal. Mach. Intell., vol. 12, pp. 629-639, 1990.

[8] V. Caselles, R. Kimmel, and G. Sapiro, "Geodesic Active Contours," International Journal of Computer Vision, vol. 22, pp. 61-79, 1997.

[9] S. Osher and J. A. Sethian,"Front propagating with curvature dependent speed: Algorithms based on Hamilton Jacobi formulation," Journal of Computational Physics, vol. 79, pp. 1249, 1988.

[10] L. Ibánez, W. Schroeder, L. Ng, and B. J. Cates, The ITK Software Guide, 2nd ed, 2005.

[11] W. Schroeder, K. Martin, and B. Lorensen, The Visualization Toolkit: An Object-Oriented Approach To 3D Graphics, 2nd ed., Prentice Hall, 1997.

[12] M. Campana., "A Framework for 4D-Biomedical Image Process- ing, Visualization and Analysis," 29th Annual International Confer- ence of the IEEE Engineering in Medicine and Biology Society.

[13] J. D. Ballard,"Generalizing the Hough Transform to Detect Arbitrary Shapes," Pattern Recognition, vol. 13, pp. 111-122, 1981.

[14] C. Melani.,"Tracking cells in a live zebrafish embryo," 29th Annual International Conference of the IEEE Engineering in Medicine and Biology Society. 